\title{
Serial Histological Sectioning of the Intact Mouse Hind Paw for Quantification of the Anatomical Distribution of Meissner-like Corpuscles across the Skin
}

\author{
Valerie Wai ${ }^{1}$, Matthew Vickaryous ${ }^{2}$, Leah Bent ${ }^{1}$ and Andrea L. Clark ${ }^{*}$
}

*Correspondence: alclark@uoguelph.ca

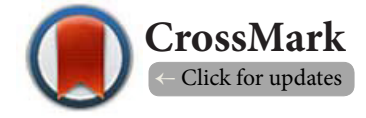

'Department of Human Health and Nutritional Sciences, College of Biological Science, University of Guelph, Guelph, ON N1G2W1, Canada. ²Department of Biomedical Sciences, Ontario Veterinary College, University of Guelph, Guelph, ON N1G2W1, Canada.

\begin{abstract}
Background: Mechanoreceptors at the terminal ends of afferent nerves in the foot sole are critical for gait and balance. While previous studies have mapped afferent receptive fields and innervation densities across the foot sole using microneurography, the location and distribution of mechanoreceptors in the foot sole skin is unknown. In humans, histological investigations are limited to specimens obtained during amputation or embalmed tissues. These can be difficult to obtain and the latter poorly preserved at the extremity of the foot sole. Mice serve as an appropriate animal model to study mechanoreceptors whose function is conserved across mammalian species, however serial sectioning of intact mouse hind paws is challenging due to the dense bones and variety of soft tissues present. Consequently, digits and footpads are typically isolated for analysis and inferences made regarding mechanoreceptor density across the whole hind paw. There are no published protocols for serial histological sectioning of mouse hind paws and attempts at serially sectioning intact rat hind paws have been unsuccessful.
\end{abstract}

Methods: We conducted eight experiments optimizing tissue preparation, chemical processing and sectioning techniques to achieve serial sectioning and staining of intact mouse hind paws. Two additional approaches (isolated hind paw skin and footpad biopsies) were compared to the intact hind paw approach to determine the optimal method for mechanoreceptor visualization and localization.

Results: The optimized protocol for serial sectioning of intact hind paws included five days of fixation in $4 \%$ paraformaldehyde, ten days of decalcification in Cal-Ex ${ }^{\mathrm{TM}}$ II, a 9-hour automated tissue processing protocol, embedding in a special formulated paraffin, and sectioning with specific techniques. Of the three approaches, intact hind paws provided the most context to structures visualized in the hind paw skin and thus, was the recommended protocol for future studies. Meissner-like corpuscles were located in the footpads and most abundantly in the footpads adjacent to digits II to $\mathrm{V}$ of the hind paw.

Conclusion: The method for serial sectioning of the intact hind paws outlined in this study will allow future analysis of mechanoreceptor distribution and density in transgenic or disease mouse models.

Keywords: Histology, serial sectioning, mouse, hind paws, decalcification, Meissner's corpuscles, mechanoreceptors, fixation, tissue processing

\section{Introduction}

The skin forms a barrier with the surrounding environment, protecting the body from chemical, mechanical and thermal exposure and enabling the sensation of heat, cold, pain and touch [1-3]. Mammalian glabrous skin is specialized for dis- criminative touch and mobility, enabling controlled movements during gait and balance $[2,4]$. Critical to this function are afferent units, consisting of an afferent sensory neuron and a mechanoreceptor at its terminus in the dermis or epidermis. Each mechanoreceptor type responds to specific skin stretch

(ㄷ) 2020 Clark et al; licensee Herbert Publications Ltd. This is an Open Access article distributed under the terms of Creative Commons Attribution License (http://creativecommons.org/licenses/by/3.0). This permits unrestricted use, distribution, and reproduction in any medium, provided the original work is properly cited. 
and/or pressure stimuli, which are then transduced into action potentials and carried by their associated afferent neurons to the central nervous system [2,3]. Afferent neurons are classified based on their firing characteristics (rapidly adapting (RA) or slowly adapting (SA)) and the size of their receptive field area (small (I) or large (II)) [5,6]. RAI afferents terminate as Meissner's corpuscles in the dermal papillae [6]. Meissner's corpuscles are imperative for minute skin indentations and grip control [6]. RAll afferents terminate as Pacinian corpuscles deep in the dermis, which detect and transmit vibratory stimuli [6]. SAI afferents terminate as Merkel cells in the stratum basale of the epidermal ridges and detect sustained skin indentation and textural changes. Lastly, SAll afferents terminate as Ruffini endings, responding to stretching of the skin [6].

To date, Meissner-like corpuscles (RAI) and Merkel cells (SAI) have been found in mouse hind paw skin [2,7] and Pacinian corpuscles (RAll) have been located in the interosseous membranes and deep tissues adjacent to bones in the forepaw and hind paw [8-10]. Ruffini endings (SAll) have yet to be identified in mice despite positive SAll neural response recordings in an ex vivo skin-nerve preparation [10]. These observations of mechanoreceptors in the mouse have utilized forelimbs or isolated footpads and interosseous membranes from the hind and fore paws [7,11-13]. As such, the anatomical location and density of mechanoreceptors across the entirety of the hind paw is still unknown.

Current knowledge about mechanoreceptors and their responses in the foot sole has been largely derived using microneurography $[4,5,14]$. This is an in vivo technique carried out in humans that involves the insertion of a microelectrode into a peripheral nerve to record neural activity in response to a stimulus [4]. Similar in vivo and ex vivo approaches to record responses from single sensory fibres have also been developed in mice to map single afferent centers in the glabrous hind paw skin $[15,16]$. Single afferent recordings, however, may not necessarily represent the sensory response of a single mechanoreceptor. Single afferent recordings may arise from the combined responses of multiple terminal branches of a single afferent that each, presumably, end in a mechanoreceptor [17-19]. It is also possible that some mechanoreceptors are served by multiple afferents $[15,18]$. Thus, direct visualization of the hind paw skin using a histological approach is necessary to determine the anatomical location and density of mechanoreceptors across the entire foot sole.

In humans, serial histological sectioning and staining is limited to tissues that are excised and biopsied during surgery, such as amputations due to vascular pathology, or cadaveric tissues. While freshly excised and biopsied surgical tissues can be perfectly preserved in formaldehyde, peripheral skin tissues of embalmed cadavers are often poorly penetrated by fixative. In contrast, mice and their tissues are more readily available, present a similar biology to humans and allow for tissue specific gene deletion that can probe the underlying mechanisms of mechanoreceptor function [20-23]. Previous histological and microscopy studies of the mouse hind paw typically section and image isolated footpads or digits. As such, studies often infer about mechanoreceptor density across the entire hind paw based only on isolated histology in the pads and digits $[13,15,24]$. To fully understand how the distribution of mechanoreceptors across the foot sole correlates to its neurological responses, the hind paw glabrous skin must be fully visualized microscopically through serial sectioning.

We are not aware of any published methods on serial sectioning of the mouse hind paw. Serial sectioning of the intact rat hind paw however has proved 'impossible' due to insufficient penetration of solutions during fixation and decalcification [25]. Thus, despite various alterations in tissue dissection, fixation and decalcification, rat hind paws were poorly processed and could not be serially sectioned on the microtome [25]. The purpose of this study therefore was to develop a protocol for serial histological sectioning of the mouse hind paw to enable visualization and anatomical mapping of mechanoreceptors across the plantar surface. To this end mouse hind paw dissection, tissue preparation, chemical processing, sectioning and staining were optimized.

\section{Materials and Methods \\ Ethics and mice}

All animal procedures were approved by the Animal Care Committee at the University of Guelph (AUP\#3960). Ten experiments were conducted, each utilizing the left and right hind paw of three wildtype BALB/c mice. Mice were 4-6 months old and skeletally mature. To minimize blood flow during micro-dissection, mice were anesthetized (isoflurane) and euthanized by cardiac puncture followed by cervical dislocation.

\section{Dissection and tissue preparation Hind paw skin}

To visualize and map mechanoreceptors throughout the skin while removing the need for bone decalcification, our first approach was to isolate the hind paw skin from the mouse. The hind paw was isolated from the mouse by a transverse cut to the distal tibia/fibula using a razor blade and pinned down at the tibialis anterior muscle with the dorsal foot facing up. The glabrous skin was isolated under a dissection microscope (Leica M60, Wetzlar, Germany) by making cuts on the dorsal and plantar skin (Figures 1A, 1B) with a scalpel and then gently pulling the skin distally. To keep the skin flat during processing it was placed on one side of a small piece of cardstock $(2.5 \mathrm{~cm} \times 3 \mathrm{~cm})$ with the subcutaneous fat side facing downwards. The cardstock was then folded over on top of the skin and its edges stapled together.

\section{Intact hind paws}

To visualize and map mechanoreceptors in the skin with added context to their locations relative to the bones of the hind paw, intact hind paws were histologically processed. Hind paws were first coated with the chemical depilator $\mathrm{Nair}^{\mathrm{TM}}$ for five 


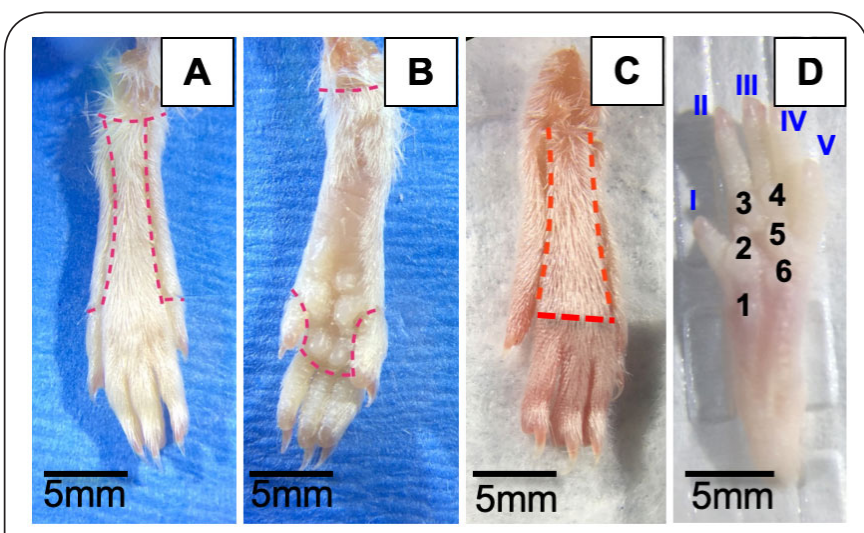

Figure 1. Cuts (red dashed line) made to the (A) dorsal and (B) plantar foot for experiments using isolated glabrous hind paw skin, and (C) the dorsal skin for removal for experiments using the intact hind paw. (D) Digits I to V and footpads 1-6 on the plantar hind paw.

minutes and then rinsed thoroughly with water. The intact hind paw was then isolated from the mouse by a transverse cut at the articulation of the tibia and the intermedium using a razor blade [26]. Nails were removed by trimming the nail to the nail bed using surgical scissors. For samples with dorsal skin removed, three cuts were made along the dorsal skin (Figure 1C). The dorsal skin was then pulled distally and removed to improve access of reagents to the tendons and musculature beneath. In experiment eight where ethylenediaminetetraacetic acid (EDTA) was employed, hind paws were bisected either in the sagittal or coronal planes using a razor blade to improve reagent penetration into the hind paw. For the sagittal bisection, the first three digits constituted one half, and the fourth and fifth digits the other half. For the coronal bisection, the cut was made halfway between the anterior border of the digits and the heel.

\section{Footpad biopsy}

To visualize mechanoreceptors specifically in the footpads without additional context to the remainder of the hind paw, footpad biopsies were histologically processed. The sacrificed mouse was placed supine and a $1 \mathrm{~mm}$ diameter skin biopsy punch tool (VWR, Mississauga, ON) was used to extract each of the six footpads (Figure 1D). Kimwipes ${ }^{\mathrm{TM}}(4 \times 5 \mathrm{~cm})$ and gauze were soaked with phosphate buffered saline (PBS) to keep the biopsy punches from dehydrating and each footpad biopsy was wrapped in a Kimwipe ${ }^{\mathrm{TM}}$ and then wrapped in gauze and placed into a tissue cassette. The Kimwipe ${ }^{\mathrm{TM}}$ and gauze kept the footpad biopsies hydrated during handling and provided a penetrable cushion within the cassette throughout tissue processing.

\section{Chemical processing}

All tissues were fixed in 4\% paraformaldehyde (PFA) for 18 hours -5 days (Table 1). Following fixation, intact hind paws were decalcified using Cal-Ex ${ }^{\mathrm{TM}}$ I, Cal-Ex ${ }^{\mathrm{TM}}$ II (Fisher Scientific, Whitby, ON) or EDTA for 4 days - 14 weeks (Table 1). Fixation and decalcification were carried out on a rocking platform at room temperature and decalcification reagents were replaced every 2-4 days. To ensure enough reagent was present for complete processing, the volume of all chemicals used was at least 20 times the tissue volume. As exposure to fixation and decalcification reagents can negatively impact tissue antigenicity, our goal was to enable serial sectioning of the tissues while minimizing these processing times $[25,27,28]$.

Tissue decalcification was assessed periodically by a chemical test or, if this was not possible, a mechanical test. The chemical test consisted of combining $5 \mathrm{~mL}$ of the Cal-Ex II solution from the sample tubes with $10 \mathrm{~mL}$ of a 1:1 solution of ammonium oxalate and ammonium hydroxide. A precipitate in the combined solution indicated incomplete decalcification. Alternatively, a mechanical test was performed by repeatedly cutting the hind paw in the coronal plane from anterior to posterior. The hardness of the bone was assessed in comparison to a non-decalcified hind paw or a decalcified paw as assessed by the chemical approach.

Following decalcification, tissues were washed in running tap water for 30 minutes and soaked in deionized water for an hour. Some samples were post-fixed in 4\% PFA for 1-6 hours while others were placed directly into $70 \%$ alcohol (reagent grade alcohol for histology, VWR, Mississauga, ON) in preparation for dehydration. Tissues were dehydrated in a series of alcohols before being cleared in xylene and infiltrated with paraffin. A general-purpose paraffin (melting point $55-57^{\circ} \mathrm{C}$, TissuePrep, Fisher Scientific, Whitby, ON)) or a 1:1 IM/LP Histoplast paraffin mix better suited for dense tissues (melting point $50-57^{\circ} \mathrm{C}$, Fisher Scientific, Whitby, ON) was used (Table 1). These final stages of processing were conducted manually or by an automated tissue processor (Pearl, Leica Biosystems Inc, Concord, ON) run on a 9 or 12-hour protocol (Tables 1 and 2).

\section{Sectioning and staining}

Blocks were sectioned $(7-8 \mu \mathrm{m})$ using a microtome (Leica RM2235, Wetzlar, Germany), with the long axis of the foot oriented either parallel or perpendicular to the blade. General purpose microtome blades (Fisher Scientific, Whitby, ON) or thicker blades specifically designed for harder tissues (Leica Biosystems Inc, Concord, ON) were used.

Due to the tendency of sections to curl or wrinkle when the tissue block was warm, and to tear or crumble when the block was dehydrated, two methods were employed to cool and hydrate the paraffin block during sectioning. First, the paraffin block was placed face down onto a large block of ice 30 minutes - 1 hour prior to sectioning. A layer of deionized water was poured onto the ice block to prevent tissue blocks from adhering to the ice and to keep them hydrated. The tissue blocks were placed back onto the ice block intermittently between sectioning. The second method involved wrapping shaved ice in gauze and securing it to the cutting surface of 
Wai et al., Journal of Histology \& Histopathology 2020,

http://www.hoajonline.com/journals/pdf/2055-091X-7-1.pdf

doi: $10.7243 / 2055-091 X-7-1$

Table 1. Comparison of tissue preparation and processing modifications for the $\mathbf{1 0}$ experiments in the study. Hair, nails or dorsal skin were intact $(\checkmark)$ or removed $(X)$. Samples were submerged in $70 \%$ alcohol $(\dagger)$ or $4 \%$ paraformaldehyde $\left(^{*}\right)$ prior to the start of the automated tissue processor protocol.

\begin{tabular}{|c|c|c|c|c|c|c|}
\hline Experiment & & $\begin{array}{l}\text { Tissue } \\
\text { preparation }\end{array}$ & $\begin{array}{l}\text { Fixation } \\
\text { (4\% PFA) }\end{array}$ & Decalcification & $\begin{array}{l}\text { Automated tissue } \\
\text { processing time }\end{array}$ & Paraffin wax \\
\hline Hind paw skin & & $\begin{array}{l}\text { X Hair } \\
\text { X Nails } \\
\text { X Dorsal Skin }\end{array}$ & $18 \mathrm{~h}$ & $\mathrm{~N} / \mathrm{A}$ & $\begin{array}{l}\text { Manual tissue } \\
\text { processing }\end{array}$ & General Purpose \\
\hline \multirow[t]{8}{*}{ Intact hind paw } & 1 & $\begin{array}{l}\sqrt{ } \text { Hair } \\
\sqrt{\text { Nails }} \\
\sqrt{ } \text { Dorsal Skin }\end{array}$ & $24 \mathrm{~h}$ & $\begin{array}{l}4 \text { days } \\
\text { Cal-Ex }{ }^{\mathrm{m}} \mathrm{I}\end{array}$ & $12 \mathrm{~h} \dagger$ & \\
\hline & 2 & $\begin{array}{l}\sqrt{ } \text { Hair } \\
\sqrt{ } \text { Nails } \\
\text { XDorsal Skin }\end{array}$ & $24 \mathrm{~h}$ & $\begin{array}{l}4 \text { days } \\
\text { Cal-Ex }{ }^{\mathrm{men}} \mathrm{I}\end{array}$ & $9 \mathrm{~h} \dagger$ & \\
\hline & 3 & $\begin{array}{l}\sqrt{ } \text { Hair } \\
\sqrt{ } \text { Nails } \\
\text { XDorsal Skin }\end{array}$ & $20 \mathrm{~h}$ & $\begin{array}{l}4 \text { days } \\
\text { Cal-Ex }{ }^{\mathrm{m}} \mathrm{I}\end{array}$ & $9 \mathrm{~h} \dagger$ & \\
\hline & 4 & $\begin{array}{l}\checkmark \text { Hair } \\
\sqrt{ } \text { Nails } \\
\text { XDorsal Skin }\end{array}$ & $24 \mathrm{~h}$ & $\begin{array}{l}25 \text { days } \\
\text { Cal-Ex }{ }^{\text {ma }} \text { I }\end{array}$ & $9 \mathrm{~h} \dagger$ & \\
\hline & 5 & $\begin{array}{l}\checkmark \text { Hair } \\
\sqrt{ } \text { Nails } \\
\text { XDorsal Skin }\end{array}$ & $\begin{array}{l}24 \text { days }+4 \text { days } \\
\text { post decalcification }\end{array}$ & $\begin{array}{l}36 \text { days } \\
\text { Cal-Ex }{ }^{\text {me }} I\end{array}$ & $9 \mathrm{~h} \dagger$ & 1:1 IM/LP Mix \\
\hline & 6 & $\begin{array}{l}\text { X Hair } \\
\text { X Nails } \\
\text { X Dorsal Skin }\end{array}$ & 5 days & $\begin{array}{l}\text { 4/9/11/15/22 } \\
\text { days Cal-Ex }{ }^{\mathrm{rs}} \text { II }\end{array}$ & $9 \mathrm{~h} \dagger$ & \\
\hline & 7 & $\begin{array}{l}\text { X Hair } \\
\text { X Nails } \\
\text { X Dorsal Skin }\end{array}$ & 5 days & $\begin{array}{l}\text { 10/12/16 days } \\
\text { Cal-Ex }{ }^{\text {su }} \text { II }\end{array}$ & $9 \mathrm{~h}^{*}$ & \\
\hline & 8 & $\begin{array}{l}\text { X Hair } \\
X \text { Nails } \\
X \text { Dorsal Skin } \\
\checkmark \text { Bisected }\end{array}$ & 5 days & $\begin{array}{l}\text { 12/13/14 weeks } \\
\text { EDTA }\end{array}$ & $9 h^{*}$ & \\
\hline Footpad biopsy & & $\begin{array}{l}\text { X Hair } \\
\text { X Nails } \\
\text { X Dorsal Skin }\end{array}$ & $24 \mathrm{~h}$ & N/A & $9 h^{*}$ & \\
\hline
\end{tabular}

Table 2. Comparison of the nine- and twelve-hour tissue processing protocols used. ${ }^{\star} 4 \%$ paraformaldehyde (PFA) was substituted for $70 \%$ alcohol for experiments 7,8 (intact hind paws) and footpad biopsy processing.

\begin{tabular}{llll}
\hline \multicolumn{2}{c}{ 9h Automated Tissue Processing } & \multicolumn{2}{c}{ 12h Automated Tissue Processing } \\
Protocol & & \multicolumn{1}{c}{ Protocol } \\
\hline Reagent & Time & Reagent & Time \\
\hline $70 \%$ alcohol or & $5-6 \mathrm{~h}$ & $4 \%$ PFA (manual) & $1 \mathrm{~h}$ \\
$4 \% \mathrm{PFA}^{*}$ & & & \\
$70 \%$ alcohol & $45 \mathrm{~min}$ & $70 \%$ alcohol & $4 \mathrm{~h}$ \\
$90 \%$ alcohol & $45 \mathrm{~min}$ & $70 \%$ alcohol & $1 \mathrm{~h}$ \\
$95 \%$ alcohol & $45 \mathrm{~min}$ & $95 \%$ alcohol & $1 \mathrm{~h}$ \\
$100 \%$ alcohol & $45 \mathrm{~min}$ & $95 \%$ alcohol & $1 \mathrm{~h}$ \\
$100 \%$ alcohol & $45 \mathrm{~min}$ & $100 \%$ alcohol & $1 \mathrm{~h}$ \\
$100 \%$ alcohol & $45 \mathrm{~min}$ & $100 \%$ alcohol & $1 \mathrm{~h}$ \\
Xylene & $45 \mathrm{~min}$ & $100 \%$ alcohol & $1 \mathrm{~h}$ \\
Xylene & $45 \mathrm{~min}$ & Xylene & $1 \mathrm{~h}$ \\
Xylene & $45 \mathrm{~min}$ & Xylene & $1 \mathrm{~h}$ \\
Paraffin infiltration $\left(62^{\circ} \mathrm{C}\right)$ & $45 \mathrm{~min}$ & Paraffin infiltration $\left(62^{\circ} \mathrm{C}\right)$ & $2 \mathrm{~h}$ \\
Paraffin infiltration $\left(62^{\circ} \mathrm{C}\right)$ & $45 \mathrm{~min}$ & Paraffin infiltration $\left(62^{\circ} \mathrm{C}\right)$ & $2 \mathrm{~h}$ \\
Paraffin infiltration $\left(62^{\circ} \mathrm{C}\right)$ & $45 \mathrm{~min}$ & - & \\
\hline
\end{tabular}


Wai et al., Journal of Histology \& Histopathology 2020,

the tissue block using rubber bands. The shaved ice packet was applied for 30 mins - 1 hour prior to sectioning and intermittently when needed during sectioning.

Serial sections were floated onto glass slides (Superfrost Plus, Fisher Scientific, Whitby, ON) with four sections on each slide. Sectioning a mouse hind paw from the most medial to the most lateral aspect in this way resulted in approximately 100 slides. Select slides were stained by hand using hematoxylin and eosin (H\&E) or hematoxylin, fast green and safranin-O. These staining protocols were adopted from previous studies with some modifications $[20,29]$. Briefly, H\&E stained sections were submerged in hematoxylin, differentiated in a solution of $1 \%$ hydrochloric acid in $70 \%$ alcohol and blued using $0.2 \%$ ammonia water. Hematoxylin, fast green and safranin-O stained sections were submerged in hematoxylin, differentiated and blued as stated above, submerged in $0.2 \%$ fast green, dipped in $1 \%$ acetic acid and submerged in $0.1 \%$ Safranin-O. A modified Harris' hematoxylin (Fisher Scientific, Whitby, ON) was used for both protocols and all slides were mounted with coverslips using Cytoseal ${ }^{\mathrm{TM}} 60$ (Thermo Scientific, Burlington, ON).

\section{Visualization of Meissner-like Corpuscles}

Two slides from the center of each digit were selected. One slide was stained using H\&E and the other using hematoxylin, fast green and safranin-O to determine which stain would allow the best visualization of Meissner-like corpuscles. Different regions of the foot were identified on each slide (Table 3). Meissner-like corpuscles were counted in these different regions of the hind paw on all four sections of each slide using light microscopy (100X 1.25 N.A. oil immersion lens, Nikon Eclipse E400 microscope) by one observer. Meissnerlike corpuscles in mouse skin range between 10-30 $\mu \mathrm{m}$ in size [7] while each section on a slide was $8 \mu \mathrm{m}$ thick, therefore receptors were only counted once if they were in a similar position across three consecutive sections to avoid double counting. The number of Meissner-like corpuscles observed in the different regions of the foot on the two slides from the center of each digit were summed. An average number of Meissner-like corpuscles across three different mice was then calculated for each region of the foot.

\section{Results}

\section{Hind Paw Skin}

Isolation of the glabrous hind paw skin from the surrounding hard tissues of the hind paw simplified tissue processing, because decalcification steps were not required. The cardstock method prevented the isolated hind paw skin from curling during manual tissue processing. During sectioning however, the tissue often crumbled, curled in on itself and appeared dry. The ice block method improved sectioning temporarily for 4-5 sections, but this was inconsistent between blocks. Consequently, serial sectioning across the hind paw skin was unattainable. Of the sections that were collected, H\&E staining revealed the different layers in the skin including the stratum corneum, epidermis and dermis (Figure 2). Meissner-like corpuscles were seen in the dermal papillae of the footpads,

Table 3. The number of Meissner-like corpuscles and their location across the plantar hind paw surface observed from two stained slides taken from the center of each digit. A cartoon of a sagittal foot cross-section indicates the specific locations. '-' indicates location not present on the slides.

\begin{tabular}{|c|c|c|c|c|c|c|c|}
\hline \multirow[t]{2}{*}{ Digit } & \multirow[t]{2}{*}{ Foot Pad } & \multicolumn{6}{|c|}{ Meissner Count } \\
\hline & & Heel (A) & $\begin{array}{c}\text { Plantar } \\
\text { metatarsal } \\
\text { skin (B) }\end{array}$ & $\begin{array}{c}\text { Footpads } \\
\text { (C) }\end{array}$ & $\begin{array}{l}\text { Plantar } \\
\text { phalanges } \\
\text { (D) }\end{array}$ & $\begin{array}{c}\text { Distal tip } \\
\text { of digit } \\
\text { (E) }\end{array}$ & $\begin{array}{c}\text { Dorsal } \\
\text { phalanges } \\
\text { (F) }\end{array}$ \\
\hline 1 & - & 0 & 0 & - & 0 & 0 & 0 \\
\hline 2 & - & 0 & 0 & - & 0 & 0 & 0 \\
\hline \multirow{2}{*}{3} & 2 & \multirow{2}{*}{0} & \multirow{2}{*}{0} & 2 & \multirow{2}{*}{0} & \multirow{2}{*}{0} & \multirow{2}{*}{0} \\
\hline & 3 & & & 12.5 & & & \\
\hline \multirow{2}{*}{4} & 4 & \multirow{2}{*}{0} & \multirow{2}{*}{0} & 8 & \multirow{2}{*}{0} & \multirow{2}{*}{0} & \multirow{2}{*}{0} \\
\hline & 5 & & & 10.5 & & & \\
\hline 5 & 5 & 0 & 0 & 0 & 0 & 0 & 0 \\
\hline
\end{tabular}


but not in other areas of the skin (Figure 2). While visualization of Meissner-like corpuscles was possible, this approach presented challenges in orientation during analysis as it was difficult to identify the location of the footpads relative to the rest of the hind paw, and even more challenging to differentiate between the six footpads.

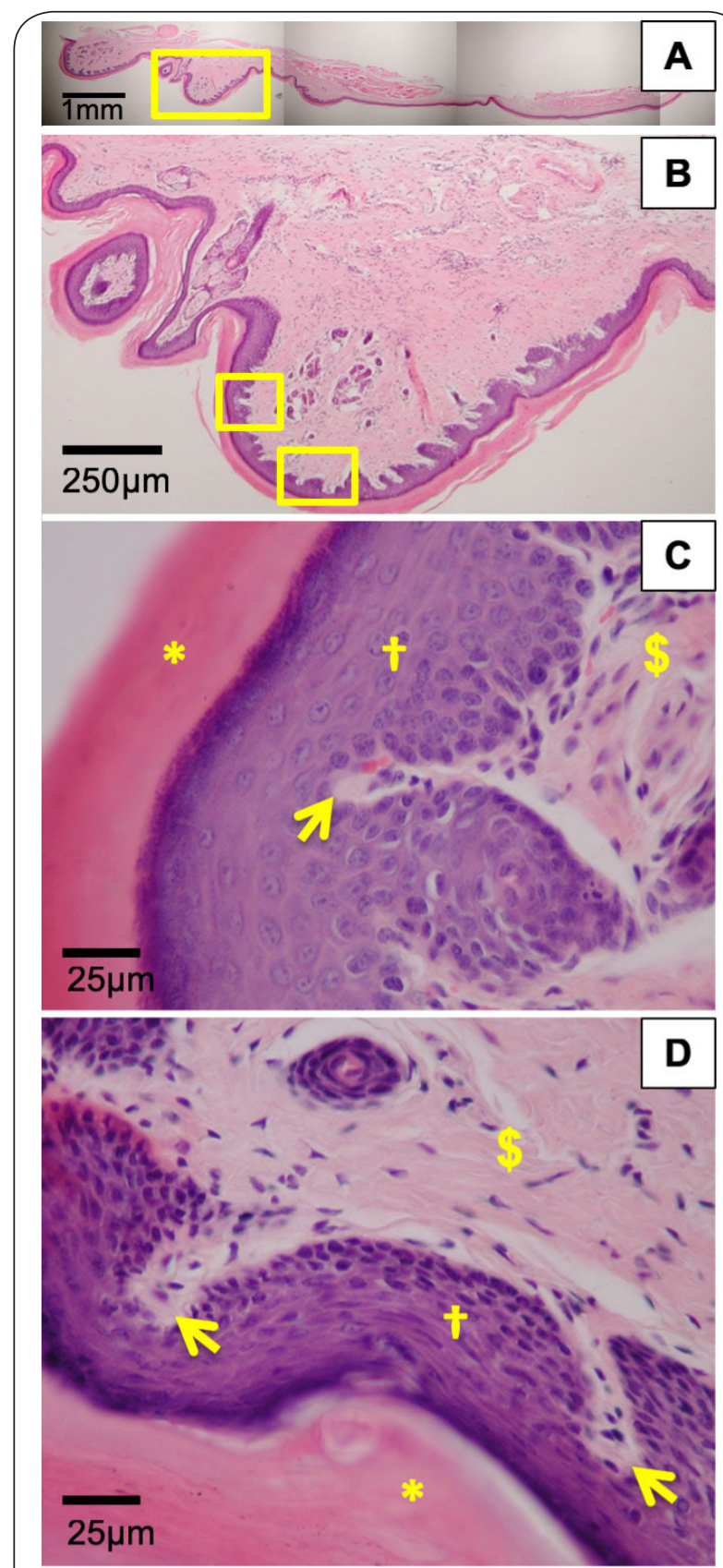

Figure 2. Light microscopy images of the glabrous hind paw skin containing Meissner-like corpuscles (yellow arrows). The boxed area in $\mathbf{A}$ is magnified in $\mathbf{B}$, and the boxed areas in $\mathbf{B}$ are magnified in $\mathbf{C}$ and $\mathbf{D}$. Sagittal sections $(7 \mu \mathrm{m})$ stained with hematoxylin and eosin. ${ }^{*}$ stratum corneum, $\dagger$ epidermis, $\$$ dermis.

\section{Intact Hind Paws}

The intact hind paws provided full context to the location of mechanoreceptors. Since intact hind paws contain a variety of tissue types (skin, muscle, connective tissue, and bone), each with its unique texture and hardness, extensive experimentation with tissue preparation, fixation, decalcification, processing and sectioning techniques were required.

\section{Tissue preparation}

Removal of hair using a depilator increased adhesion between the paraffin and the tissue during sectioning, making the sections easier to transport from the microtome to the water bath ready to be picked up on microscope slides. Nail trimming prevented large pieces of nail from chipping away from the paraffin block during sectioning causing the tissue to tear and subsequent loss of section integrity. Removing the dorsal skin improved reagent penetration during fixation, decalcification and tissue processing, resulting in fewer white dry patches in the tissues during sectioning and thus improved section quality. Interestingly, bisected hind paws in both sagittal and coronal planes showed a loss of tissue integrity and higher separation between tissue layers.

\section{Fixation and processing}

Tissue morphology of the intact hind paw was improved as fixation (4\% PFA) time was increased from 20 to 24 hours (Figure 3). Further extension of fixation time to five days had no effect on H\&E staining and produced consistently highquality sections of morphologically preserved tissues. A 12-hour

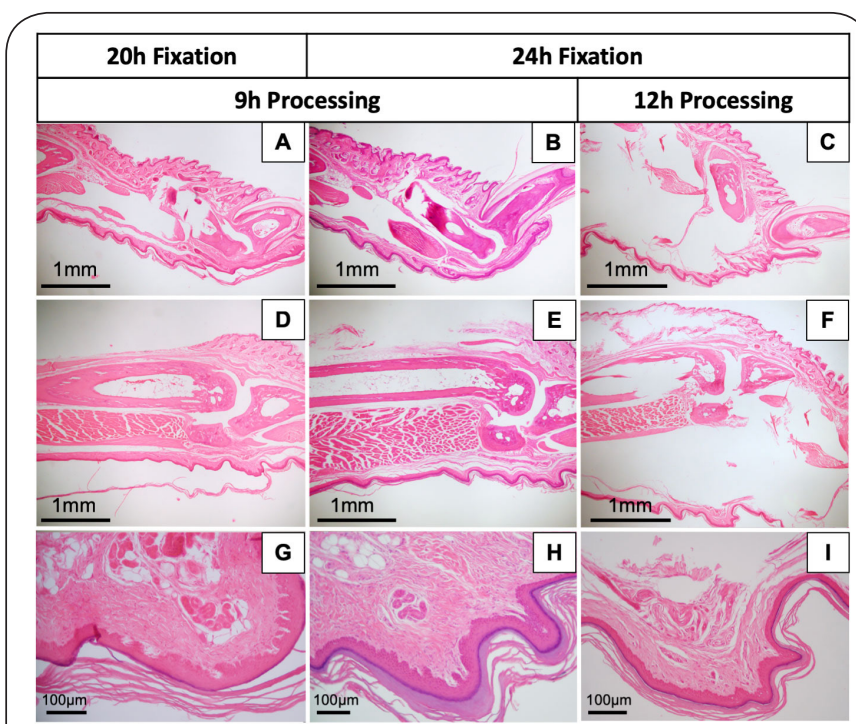

Figure 3. Light microscopy images of intact hind paws (toe (A,B,C), metatarsal (D,E,F), footpad $(\mathbf{G}, \mathbf{H}, \mathbf{I}))$ fixed ( $4 \%$ PFA) for 20 or 24 hours, and processed (automated) using a 9 or 12 hour protocol. Sagittal sections $(7 \mu \mathrm{m})$ stained with hematoxylin and eosin. Note the improved preservation of tissue morphology and integrity throughout the hind paw with longer fixation and shorter processing times $(\mathbf{B}, \mathbf{E}, \mathbf{H})$. 
Wai et al., Journal of Histology \& Histopathology 2020,

automated tissue processing protocol for intact hind paws resulted in dry and brittle tissues during sectioning with separation of the skin from other anatomical structures (Figure 3). A shortened 9-hour protocol improved the hydration of tissues and maintained the relationship between the skin and deeper components of the hind paw (Figure 3). Infiltrating and embedding hind paws with the 1:1 IM/LP Histoplast paraffin mix decreased the wrinkling of sections and chipping out of bone from the block and resulted in improved cohesion between the tissue and the paraffin compared to hind paws prepared with general-purpose paraffin.

\section{Decalcification}

By day four of decalcification with Cal-Ex ${ }^{T M}$ I, both mechanical testing and chemical testing confirmed complete decalcification, however, section quality was poor. Large, opaque, white pieces of metatarsal bones (digits two to four) as well as the ankle bones often chipped from the tissue block, leaving a large hole in the section and compromising the integrity of subsequent sections. When decalcification with $\mathrm{Cal}^{-\mathrm{Ex}^{\mathrm{TM}} \text { I was }}$ extended to 25 and 36 days, sectioning was greatly improved, and the bone no longer chipped from the block. With lengthened decalcification times however, hematoxylin staining decreased significantly demonstrating nucleic acid damage but eosin staining of proteins was less affected (Figure 4).

Decalcifying with Cal-Ex ${ }^{\mathrm{TM}}$ II resulted in similar sectioning difficulties as with Cal-Ex ${ }^{\mathrm{TM}} \mathrm{I}$ at day four, with large fragments

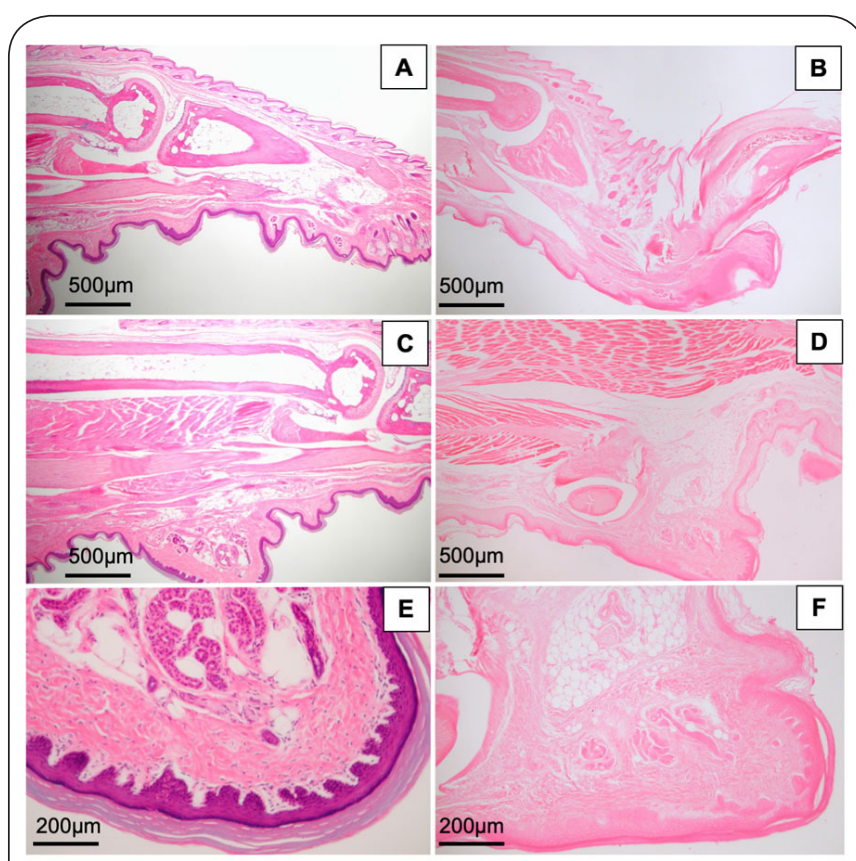

Figure 4. Light microscopy images of intact hind paws (toe $(\mathbf{A}, \mathbf{B})$, metatarsal $(\mathbf{C}, \mathbf{D})$, footpad $(\mathbf{E}, \mathbf{F}))$ decalcified in Cal-Ex ${ }^{\mathrm{Tm}}$ I for $10(\mathbf{A}, \mathbf{C}, \mathbf{E})$ or $36(\mathbf{B}, \mathbf{D}, \mathbf{F})$ days. Sagittal sections $(8 \mu \mathrm{m})$ stained with hematoxylin and eosin. Note the absence of hematoxylin staining at the 36 day time point (B,D,F). of metatarsal bone chipping from the paraffin block making serial sectioning impossible. Extending Cal-Ex ${ }^{\mathrm{TM}} \|$ decalcification time to nine and 11 days significantly decreased the frequency and size of metatarsal chips which then did not hinder the integrity of subsequent sections or affect visualization of the skin. Further extending Cal-Ex ${ }^{T M}$ II decalcification time out to 15 and 22 days resulted in limited improvement in bone chipping and tissue integrity during sectioning, however by 22 days hematoxylin staining was noticeably weaker with less effect on eosin staining (Figure 5).

Intact hind paws were also decalcified in EDTA however decalcification times were much longer $(12,13$ and 14 weeks) than for Cal-Ex ${ }^{\mathrm{TM}}$, making it the least efficient decalcifying agent.

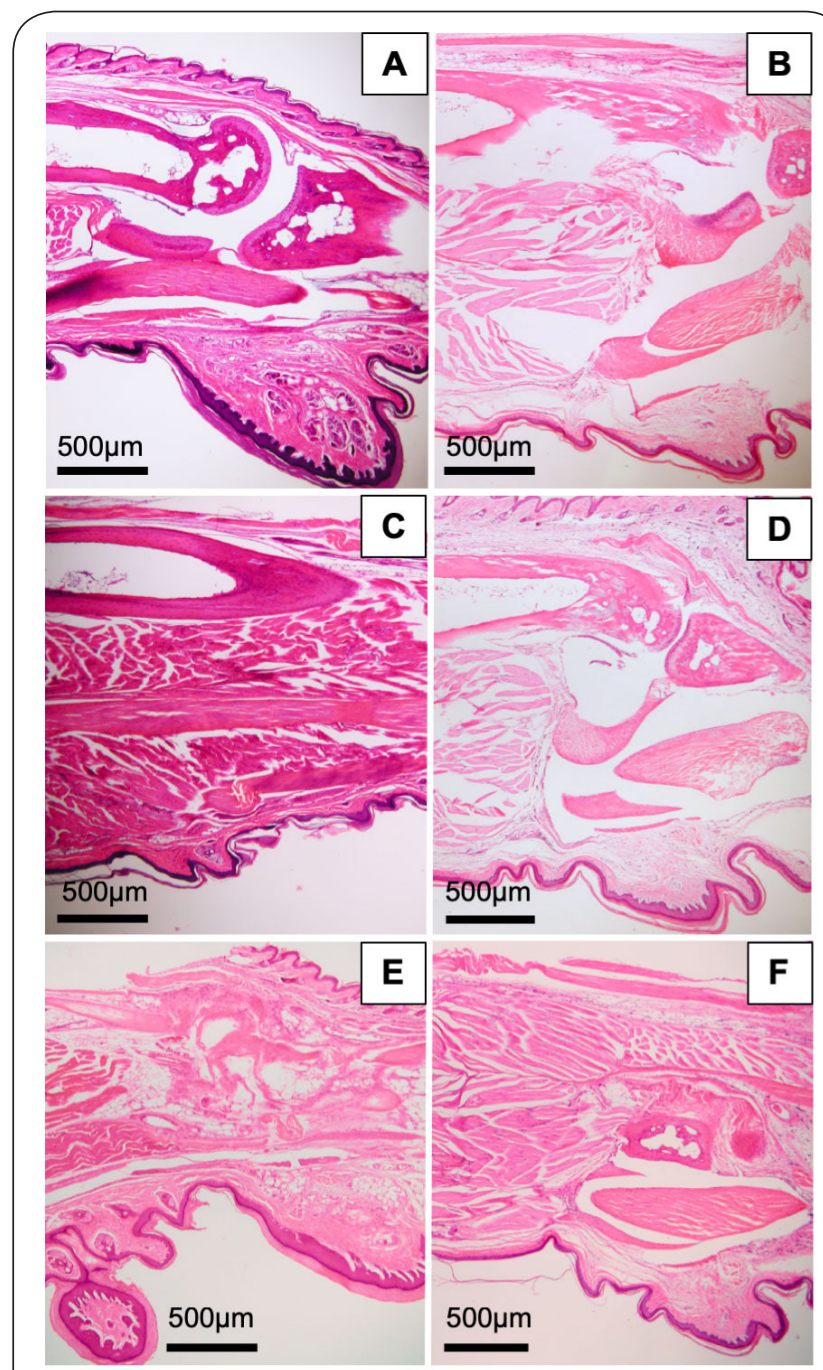

Figure 5. Light microscopy images of the metatarsal region of intact hind paws decalcified in Cal-Ex ${ }^{\text {Tx }}$ II for 9 (A), 11 (C) or 22 (E) days, or EDTA for 12 (B), 13 (D) or 14 (F) weeks. Sagittal sections $(8 \mu \mathrm{m})$ stained with hematoxylin and eosin. Note the decrease in tissue integrity with EDTA $(\mathbf{B}, \mathbf{D}, \mathbf{F})$ compared to Cal-Ex ${ }^{\mathrm{xt}}$ II $(\mathbf{A}, \mathbf{C}, \mathbf{E})$ and the reduced hematoxylin staining at later time points $(\mathbf{E}, \mathbf{F})$. 
Mechanical testing demonstrated that EDTA decalcification was complete by week 12 however sectioning revealed poor tissue morphology. The different layers of soft tissues were visibly pulling apart from one another and the skin was separating from deeper structures in the foot. Quality of hematoxylin staining was poor, comparable to that seen with $\mathrm{Cal}^{-\mathrm{Ex}^{\mathrm{TM}}} \mathrm{Il}$ at 22 days, with less effect on eosin staining (Figure 5 ).

\section{Sectioning}

Cooling the paraffin block on an ice block for 30 minutes decreased wrinkling of sections however patches of tissue in the center of the block appeared white and dry, and sectioned poorly. In contrast, cooling and hydrating the block with shaved ice wrapped in gauze decreased the occurrence of dried and crumbling tissue resulting in significantly improved sectioning.

\section{Footpad Biopsies}

Footpad biopsies allowed visualization of mechanoreceptors specifically in the footpads without additional context to the remainder of the hind paw. Tissue processing was simplified because decalcification steps were not required. Serial sectioning was successful for the footpad biopsies. H\&E stained strongly and allowed for visualization of Meissner-like corpuscles in the dermal papillae of the footpads.

\section{Visualization of Meissner-like Corpuscles in Intact Hind Paws}

Serial sections were obtained from intact hind paws fixed in 4\% PFA for five days, decalcified for ten days in Cal-Ex ${ }^{\mathrm{TM}}$ II and automatically processed for nine hours. Staining with either H\&E or hematoxylin, safranin-O and fast green enabled identification of different regions of the hind paw (Figure 6 , Table 3). Footpads one and six were not aligned in the same sectioning plane as the digits of the hind paw and thus were not visible on the slides sampled from the center of each digit. Meissner-like corpuscles were seen in the dermal papillae of the glabrous skin of the footpads (Figure 6), with footpads three, four and five having the largest numbers (Table 3 ). Meissner-like corpuscles were not present in other regions of the hind paw (Table 3) and other mechanoreceptor types (Merkel cells, Pacinian corpuscles and Ruffini endings) were not visualized.

\section{Discussion}

The purpose of this study was to develop a protocol for serial histological sectioning of the mouse hind paw that would enable visualization and anatomical mapping of mechanoreceptors across the plantar surface. To this end we used three different approaches - footpad biopsies, hind paw skin and intact hind paws and optimized the protocols to enable serial sectioning while minimizing fixation and decalcification times to best preserve antigenicity. The protocol we most highly recommend is the intact hind paw approach

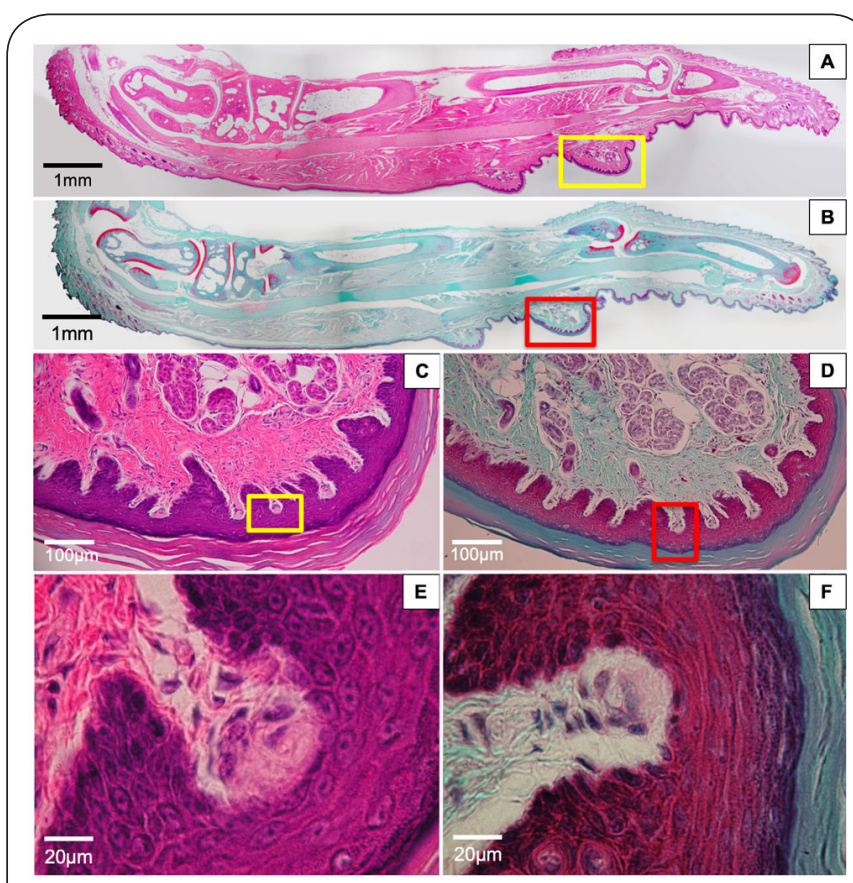

Figure 6. Light microscopy images of serial sections of intact hind paws. The boxed area in $\mathbf{A}$ and $\mathbf{B}$ is magnified in $\mathbf{C}$ and $\mathbf{D}$, and the boxed area in $\mathbf{C}$ and $\mathbf{D}$ is magnified in $\mathbf{E}$ and $\mathbf{F}$ respectively. Sagittal sections $(8 \mu \mathrm{m})$ stained with hematoxylin and eosin $(\mathbf{A}, \mathbf{C}, \mathbf{E})$ or hematoxylin, safranin- $\mathrm{O}$ and fast green $(\mathbf{B}, \mathbf{D}, \mathbf{F})$. Various regions of the foot can be identified $(\mathbf{A}, \mathbf{B})$ including the distal tip of the digit, the dorsal and plantar surfaces of the phalanges, the footpads, the plantar metatarsal skin and the heel. Note the well-preserved tissue morphology and integrity and the Meissner-like corpuscles in the dermal papillae of the footpads.

where mechanoreceptors can be visualized within the full context of the surrounding tissues. This protocol consists of the removal of hair, nails, and dorsal skin following isolation of the hind paw from the mouse, five days of fixation in $4 \%$ PFA, ten days of decalcification in Cal-Ex ${ }^{\mathrm{TM}} \mathrm{II}$, post-fixation for five hours, a nine hour automated tissue processing protocol, infiltration and embedding in a 1:1 IM/LP paraffin mix and sectioning using the gauze and shaved ice method. We then recommend the footpad biopsy approach, where although full context relative to the hind paw is not available, the original footpad location can be recorded. Furthermore, an incision can be made on one end (i.e anterior) of the biopsy at the time of harvest to allow for orientation of the biopsy following processing. The isolated hind paw skin approach would not be recommended due to the lack of context for the location of mechanoreceptors relative to the bones of the hind paw and the difficultly in distinguishing the footpads from one another. To mitigate these challenges, a combination of incisions and/or macroscopic dyes could be used to try to improve understanding of the specimen orientation. However, multiple incisions could interfere with the integrity of the isolated hind paw skin and macroscopic dyes may be 
Wai et al., Journal of Histology \& Histopathology 2020,

compromised during tissue processing and sectioning.

Meissner-like corpuscles were more easily distinguished with the contrast of $\mathrm{H} \& \mathrm{E}$ staining rather than safranin-O, fast green and hematoxylin. $\mathrm{H} \& \mathrm{E}$ serial sections of the intact hind paws enabled visualization and anatomical localization of Meissner-like corpuscles that were isolated to the footpad dermal papillae of the glabrous skin. Footpad three was found to have the greatest number of Meissner-like corpuscles and footpad two had the least. However only two slides were sampled at each digit to visualize the mechanoreceptors thus, a larger sample of slides must be analyzed to obtain an accurate count, density or distribution of Meissner-like corpuscles across the various locations of the hind paw plantar skin. No other receptor types were identified with this preparation. Our observations are consistent with others who have found Meissner-like corpuscles in the glabrous skin of the mouse $[12,15]$. Pacinian corpuscles have not been found in rodent skin however they are abundant in the interosseous membrane of the rodent ulna and fibula [12]. SAll afferent responses have been recorded electrophysiologically in mouse hairy skin, however the Ruffini endings associated with these afferents have not been found [12]. Merkel cells have also been located in the glabrous and hairy skin of the mouse $[12,15]$. Merkel cells are known to be localized to the basal layer of the epidermis and due to their similarity in size to keratinocytes in the epidermis, immunohistochemistry is required to distinguish them [12].

It is important to note that sampling relative to the digits in the intact hind paw will not necessarily yield representative sections of each footpad because the footpads do not align systematically with the digits. For example, we did not see footpads one or six on the stained sections taken from the center of each digit due to the plane of embedding. The fifth digit was embedded flat onto the mold and as a result, the plane of sectioning was not parallel to the sagittal midline of the hind paw. During analysis, it was also a challenge to distinguish the footpads from one another in the serial sections because only two footpads were visible on a section, if any. Because the length of the digits and surrounding structures are constantly changing as one moves through the serial sections, it required careful attention to determine which footpad(s) was visible in each slide. In future studies, embedding the hind paw with the sagittal midline parallel to the plane of sectioning will allow for at least three footpads to be seen on one section, and therefore all footpads should be more easily distinguished from one another in serial sections.

While intact hind paws provided the best context for the location of Meissner-like corpuscles across the plantar skin, they presented the greatest challenge for optimization of tissue preparation, chemical processing and staining. The size and density of tissue samples is known to positively correlate with the rate of tissue processing $[25,27,30]$. The volume of the mouse hind paw is at least ten times larger than its skin alone, and therefore a greater challenge for processing. Furthermore, skin acts as a defensive barrier against the environment, mediated primarily by the hydrophobic lipids in the extracellular matrix of the stratum corneum of the epidermis [31]. This barrier prevents the penetration of the reagents during tissue processing. Previously in rat intact forepaws and hind paws, complete skin removal reduced decalcification time by $28 \%$ [25]. In a similar manner, we chose to remove the dorsal skin of the mouse hind paw to improve reagent penetration into the tissues deep to the skin from at least one surface.

We found that five days of fixation in 4\% PFA was optimal for the intact mouse hind paws in our study. This fixation time may seem excessive when compared to the rule of thumb one hour per $1 \mathrm{~mm}$ of tissue, and the recommended $24 \mathrm{~h}$ fixation time for neutral buffered formalin fixed specimens [30]. Our longer fixation time significantly improved sectioning quality and consistency, and both H\&E and safranin-O, fast green and hematoxylin staining were unaffected. It is important to note however, that antigen retrieval will likely be required for some epitopes when performing immunohistochemistry on these tissues due to the protracted fixation time [30]. Specimens including bone are often embedded in plastic formulations, in order to match the consistency of the mineralized bone to the embedding medium [32]. In a similar manner, we found that sectioning of the mouse hind paw benefitted from embedding in a harder 1:1 IM/LP Histoplast paraffin mix.

EDTA is a standard decalcifying agent that results in good antigenic preservation $[25,27,33]$. In comparison to decalcifiers such as formic acid (found in Cal-Ex ${ }^{\mathrm{TM}}$ ), EDTA is a more gentle but slower chelating agent. Decalcification of rat femurs requires 21 days in 10\% EDTA versus eight days for 3-5\% nitric acid and $8 \%$ hydrochloric acid/formic acid [27], and intact rat hind paws decalcified in 10\% EDTA for 100 days were impossible to section due to substantial crumbling of the tissues [25]. We show that decalcification of mouse hind paws took at least eight times longer with 10\% EDTA compared to Cal-Ex ${ }^{\mathrm{TM}}$. Others have reported that $10 \%$ EDTA is the best decalcification agent for immunohistochemistry of rat femurs and for H\&E staining of sagittally halved and skinned rat hind paws and fore paws with/without microwave treatment $[25,27]$. For our mouse hind paws however, the protracted decalcification time in $10 \%$ EDTA caused the tissues to disintegrate and $H \& E$ staining to be compromised compared to tissues processed in Cal-Ex'

In conclusion, dissection, tissue preparation, chemical processing, sectioning techniques and staining were optimized for serial histological sectioning of the intact mouse hind paw. This protocol consists of the removal of hair, nails, and dorsal skin following isolation of the hind paw from the mouse, five days of fixation, ten days of decalcification in Cal-Ex ${ }^{\mathrm{TM}} \mathrm{II}$, postfixation for five hours, a nine hour automated tissue processing protocol, infiltration and embedding in a 1:1 IM/LP paraffin mix and sectioning using the gauze and shaved ice method. This was a first step to enable a thorough histological analysis of mechanoreceptors across the entire mouse hind paw skin, 
and their specific anatomical location and distribution. Our preliminary data are consistent with previous studies with Meissner-like corpuscles identified in the footpads of the glabrous skin while Pacinian corpuscles and Ruffini endings were absent. Merkel cells will require immunohistochemistry with antigen retrieval to distinguish them from surrounding keratinocytes. Future studies of mechanoreceptor distribution will benefit from modifications to hind paw orientation during embedding and more thorough slide sampling.

\section{Competing interests}

The authors declare that they have no competing interests.

\section{Authors' contributions}

\begin{tabular}{|l|c|c|c|c|}
\hline Authors' contributions & VW & MV & LB & ALC \\
\hline Research concept and design & -- & $\checkmark$ & $\checkmark$ & $\checkmark$ \\
\hline Collection and/or assembly of data & $\checkmark$ & -- & -- & -- \\
\hline Data analysis and interpretation & $\checkmark$ & $\checkmark$ & -- & $\checkmark$ \\
\hline Writing the article & $\checkmark$ & -- & -- & -- \\
\hline Critical revision of the article & $\checkmark$ & $\checkmark$ & $\checkmark$ & $\checkmark$ \\
\hline Final approval of article & $\checkmark$ & $\checkmark$ & $\checkmark$ & $\checkmark$ \\
\hline Statistical analysis & -- & -- & -- & -- \\
\hline
\end{tabular}

\section{Acknowledgements}

We would like to thank Jana Michaud and Jodi Morrison for their technical support and a Natural Sciences and Engineering Research Council Discovery Grant (A.C.) for financial support.

Publication history

Editor: Khin Thway, The Royal Marsden Hospital, UK.

Received: 10-Nov-2019 Final Revised: 15-Jan-2020

Accepted: 06-Feb-2020 Published: 16-Feb-2020

\section{References}

1. Yousef H, Alhajj M, and Sharma S: Anatomy, Skin (Integument), Epidermis. Treasure Island: StatPearls Publishing; 2019. Available from: http:// www.ncbi.nlm.nih.gov/pubmed/29262154

2. Zimmerman A, Bai L, and Ginty DD: The gentle touch receptors of mammalian skin. Science 2014; 346: 950-954. Available from: http://www. ncbi.nlm.nih.gov/pubmed/25414303 DOI: 10.1126/science.1254229

3. Lumpkin EA, Marshall KL, and Nelson AM: The cell biology of touch. J. Cell Biol. 2010; 191: 237-248. Available from: http://www.jcb.org/lookup/ doi/10.1083/jcb.201006074 DOI: 10.1083/jcb.201006074

4. Strzalkowski NDJ, Peters RM, Inglis JT, and Bent LR: Cutaneous afferent innervation of the human foot sole: what can we learn from singleunit recordings? J. Neurophysiol. 2018; 120: 1233-1246. Available from: https://www.physiology.org/doi/10.1152/jn.00848.2017 DOI: 10.1152/ jn.00848.2017

5. Kennedy PM and Inglis JT: Distribution and behaviour of glabrous cutaneous receptors in the human foot sole. J. Physiol. 2002; 538: 995-1002. Available from: http://doi.wiley.com/10.1113/jphysiol.2001.013087 DOI: 10.1113/jphysiol.2001.013087

6. Johnson KO: The roles and functions of cutaneous mechanoreceptors. Curr. Opin. Neurobiol. 2001; 11: 455-461. Available from: http://www. ncbi.nlm.nih.gov/pubmed/11502392 DOI: https://doi.org/10.1016/ S0959-4388(00)00234-8

7. Zelená J: Nerves and Mechanoreceptors: The Role of Innervation in the Development and Maintenance of Mammalian Mechanoreceptors. 1st ed. Cambridge: Chapman \& Hall; 1994.

8. Zelená J: The development of Pacinian corpuscles. J. Neurocytol. 1978; 7: 71-91. Available from: https://link-springer-com.subzero.lib.uoguelph.ca/ content/pdf/10.1007\%2FBF01213461.pdf DOI: 10.1007/BF01213461
9. Prsa M, Morandell K, Cuenu G, and Huber D: Feature-selective encoding of substrate vibrations in the forelimb somatosensory cortex. Nature 2019; 567: 384-388. Available from: http://dx.doi.org/10.1038/s41586019-1015-8 DOI: 10.1038/s41586-019-1015-8

10. Wellnitz SA, Lesniak DR, Gerling GJ, and Lumpkin EA: The Regularity of Sustained Firing Reveals Two Populations of Slowly Adapting Touch Receptors in Mouse Hairy Skin. J Neurophysiol 2010; 103: 3378-3388. Available from: www.jn.org DOI: 10.1152/jn.00810.2009

11. Albuerne M, De Lavallina J, Esteban I, Naves FJ, Silos-Santiago I, and Vega JA: Development of Meissner-like and Pacinian sensory corpuscles in the mouse demonstrated with specific markers for corpuscular constituents. Anat. Rec. 2000; 258: 235-242. Available from: http://doi. wiley.com/10.1002/\%28SICI\%291097-0185\%2820000301\%29258\%3A3 \%3C235\%3A\%3AAID-AR2\%3E3.0.CO\%3B2-M DOI: 10.1002/(SICI)10970185(20000301)258:3<235::AID-AR2>3.0.CO;2-M

12. Fleming $M S$ and Luo $W$ : The anatomy, function, and development of mammalian A $\boldsymbol{\beta}$ low-threshold mechanoreceptors. Front. Biol. (Beijing). 2013; 8: 408-420. Available from: http://link.springer.com/10.1007/ s11515-013-1271-1 DOI: 10.1007/s11515-013-1271-1

13. Navarro X, Verdú E, Wendelschafer-Crabb G, and Kennedy WR: Innervation of cutaneous structures in the mouse hind paw: A confocal microscopy immunohistochemical study. J. Neurosci. Res. 1995; 41: 111-120. Available from: http://doi.wiley.com/10.1002/jnr.490410113 DOI: 10.1002/jnr.490410113

14. Ribot-Ciscar E, Vedel JP, and Roll JP: Vibration sensitivity of slowly and rapidly adapting cutaneous mechanoreceptors in the human foot and leg. Neurosci. Lett. 1989; 104: 130-135. Available from: https://linkinghub.elsevier.com/retrieve/pii/030439408990342X DOI: 10.1016/03043940(89)90342-X

15. Walcher J, Ojeda-Alonso J, Haseleu J, Oosthuizen MK, Rowe AH, Bennett NC, and Lewin GR: Specialized mechanoreceptor systems in rodent glabrous skin. J. Physiol. 2018; 596: 4995-5016. Available from: http://www. ncbi.nlm.nih.gov/pubmed/30132906 DOI: 10.1113/JP276608

16. Cain DM, Khasabov SG, and Simone DA: Response properties of mechanoreceptors and nociceptors in mouse glabrous skin: An in vivo study. J. Neurophysiol. 2001; 85: 1561-1574. Available from: http://www.physiology.org/doi/10.1152/jn.2001.85.4.1561 DOI: 10.1152/jn.2001.85.4.1561

17. Parker AJ and Newsome WT: SENSE AND THE SINGLE NEURON: Probing the Physiology of Perception. Annu. Rev. Neurosci. 1998; 21: 227-277. Available from: http://www.annualreviews.org/doi/10.1146/annurev. neuro.21.1.227 DOI: 10.1146/annurev.neuro.21.1.227

18. A. Vega J, Lopez-Muniz A, G. Calavia M, Garcia-Suarez O, Cobo J, Otero $\mathrm{J}$, Arias-Carrion O, Perez-Pinera P, and Menendez-Gonzalez M: Clinical Implication of Meissner's Corpuscles. CNS Neurol. Disord. - Drug Targets 2012; 11: 856-868. Available from: http://www.eurekaselect.com/ openurl/content.php?genre=article\&issn=1871-5273\&volume=11\&issue =7\&spage=856 DOI: $10.2174 / 1871527311201070856$

19. Strzalkowski NDJ, Mildren RL, and Bent LR: Thresholds of cutaneous afferents related to perceptual threshold across the human foot sole. J. Neurophysiol. 2015; 114: 2144-2151. Available from: https://www.physiology.org/doi/10.1152/jn.00524.2015 DOI: 10.1152/jn.00524.2015

20. Shin SY, Pozzi A, Boyd SK, and Clark AL: Integrin $\alpha 1 \beta 1$ protects against signs of post-traumatic osteoarthritis in the female murine knee partially via regulation of epidermal growth factor receptor signalling Osteoarthr. Cartil. 2016; 24: 1795-1806. Available from: https://linkinghub.elsevier.com/retrieve/pii/S1063458416300966 DOI: 10.1016/j. joca.2016.05.013

21. O'Conor CJ, Ramalingam S, Zelenski NA, Benefield HC, Rigo I, Little D, Wu C-L, Chen D, Liedtke W, McNulty AL, and Guilak F: Cartilage-Specific Knockout of the Mechanosensory Ion Channel TRPV4 Decreases AgeRelated Osteoarthritis. Sci. Rep. 2016; 6: 1-10. Available from: http:// www.nature.com/articles/srep29053 DOI: 10.1038/srep29053

22. Haycraft CJ, Zhang Q, Song B, Jackson WS, Detloff PJ, Serra R, and Yoder BK: Intraflagellar transport is essential for endochondral bone formation. Development 2007; 134: 307-316. Available from: http://www.ncbi. nlm.nih.gov/pubmed/17166921 DOI: 10.1242/dev.02732

23. Zimmermann AS, Morrison SD, Hu MS, Li S, Nauta A, Sorkin M, Meyer 
Wai et al., Journal of Histology \& Histopathology 2020, http://www.hoajonline.com/journals/pdf/2055-091X-7-1.pdf

NP, Walmsley GG, Maan ZN, Chan DA, Gurtner GC, Giaccia AJ, and Longaker MT: Epidermal or Dermal Specific Knockout of PHD-2 Enhances Wound Healing and Minimizes Ischemic Injury. Moser M, ed. PLoS One 2014; 9: e93373. Available from: https://dx.plos.org/10.1371/journal. pone.0093373 DOI: 10.1371/journal.pone.0093373

24. Wong J, Bennett W, Ferguson MWJ, and McGrouther DA: Microscopic and histological examination of the mouse hindpaw digit and flexor tendon arrangement with 3D reconstruction. J. Anat. 2006; 209: 533 545. Available from: http://www.ncbi.nlm.nih.gov/pubmed/17005025 DOI: $10.1111 / \mathrm{j} .1469-7580.2006 .00625 . x$

25. González-Chávez SA, Pacheco-Tena C, Macías-Vázquez CE, and LuévanoFlores E: Assessment of different decalcifying protocols on Osteopontin and Osteocalcin immunostaining in whole bone specimens of arthritis rat model by confocal immunofluorescence. Int. J. Clin. Exp. Pathol. 2013; 6: 1972-1983. Available from: http://www.ncbi.nlm.nih.gov/ pubmed/24133575

26. Cook MJ: The Anatomy of the Laboratory Mouse. Ann Arbor, Michigan: Academic Press (The University of Michigan); 1965.

27. Liu H, Zhu R, Liu C, Ma R, Wang L, Chen B, Li L, Niu J, Zhao D, Mo F, Fu M, Brömme D, Zhang D, and Gao S: Evaluation of Decalcification Techniques for Rat Femurs Using HE and Immunohistochemical Staining. Biomed Res. Int. 2017; 2017: 1-6. Available from: http://dx.doi. org/10.1155/2017/9050754 DOI: 10.1155/2017/9050754

28. Begum F, Zhu W, Namaka MP, and Frost EE: A novel decalcification method for adult rodent bone for histological analysis of peripheral-central nervous system connections. J. Neurosci. Methods 2010; 187: 59-66. Available from: http://dx.doi.org/10.1016/j.jneumeth.2009.12.013 DOI: 10.1016/j.jneumeth.2009.12.013

29. Subramaniam N, Petrik JJ, and Vickaryous MK: VEGF , FGF -2 and TGF $\beta$ expression in the normal and regenerating epidermis of geckos: implications for epidermal homeostasis and wound healing in reptiles. $J$. Anat. 2018; 232: 768-782. Available from: http://www.ncbi.nlm.nih.gov/ pubmed/29417581 DOI: 10.1111/joa.12784

30. Howat WJ and Wilson BA: Tissue fixation and the effect of molecular fixatives on downstream staining procedures. Methods 2014; 70: 12-19. Available from: http://dx.doi.org/10.1016/j.ymeth.2014.01.022 DOI: 10.1016/j.ymeth.2014.01.022

31. Elias PM: Skin barrier function. Curr. Allergy Asthma Rep. 2008; 8: 299305. Available from: http://link.springer.com/10.1007/s11882-008-00480 DOI: 10.1007/s11882-008-0048-0

32. Porter A, Irwin R, Miller J, Horan DJ, Robling AG, and McCabe LR: Quick and inexpensive paraffin-embedding method for dynamic bone formation analyses. Sci. Rep. 2017; 7: 42505. Available from: http://www. nature.com/articles/srep42505 DOI: 10.1038/srep42505

33. Sanjai K, Kumarswamy J, Patil A, Papaiah L, Jayaram S, and Krishnan L: Evaluation and comparison of decalcification agents on the human teeth. J. Oral Maxillofac. Pathol. 2012; 16: 222-227. Available from: http://www.ncbi.nlm.nih.gov/pubmed/22923894 DOI: 10.4103/0973029X.99070

\section{Citation:}

Wai V, Vickaryous M, Bent L and Clark AL. Serial Histological Sectioning of the Intact Mouse Hind Paw for Quantification of the Anatomical Distribution of Meissner-like Corpuscles across the Skin.

J Histol Histopathol. 2020; 7:1.

http://dx.doi.org/10.7243/2055-091X-7-1 